Supporting Information

\title{
Switching Plasmon Driven Reaction Mechanism from Charge Transfer to Adsorbate Electronic Excitation using Surface Ligands
}

Hamed Kookhaee, Tefera E. Tesema, and Terefe G. Habteyes*

Department of Chemistry and Chemical Biology, and Center for High Technology Materials, University of New Mexico, Albuquerque, New Mexico 87131, United States

Corresponding email: habteyes@unm.edu

\section{Content}

$>$ Schematic of the experimental setup used to perform the SERS measurement (Figure S1)

> Scanning electron microscope images of methylene blue (MB) treated gold particles (Figure S2)

> Absorption spectum of MB in water and adsorbed on gold nanoislands (Figure S3)

> Possible outcomes of MB N-demethylation (Scheme S1)

D SERS spectra of MB adsorbed on S-CA and on R-CTAB at different excitation wavelengths at 0.5 $\mathrm{s}$ and $103 \mathrm{~s}$ illumination times (Figure S4)

Dormal and SERS spectra of methylene blue, azure B, azure A and thionine (Figure S5)

$>$ Intensity map of SERS spectra of MB adsorbed on R-CA (Figure S6)

$>$ Calculated Raman Spectra (Figure S7)

$>$ Change in relative peak intensity of representative modes as a function of illumination time (Figure S8) 


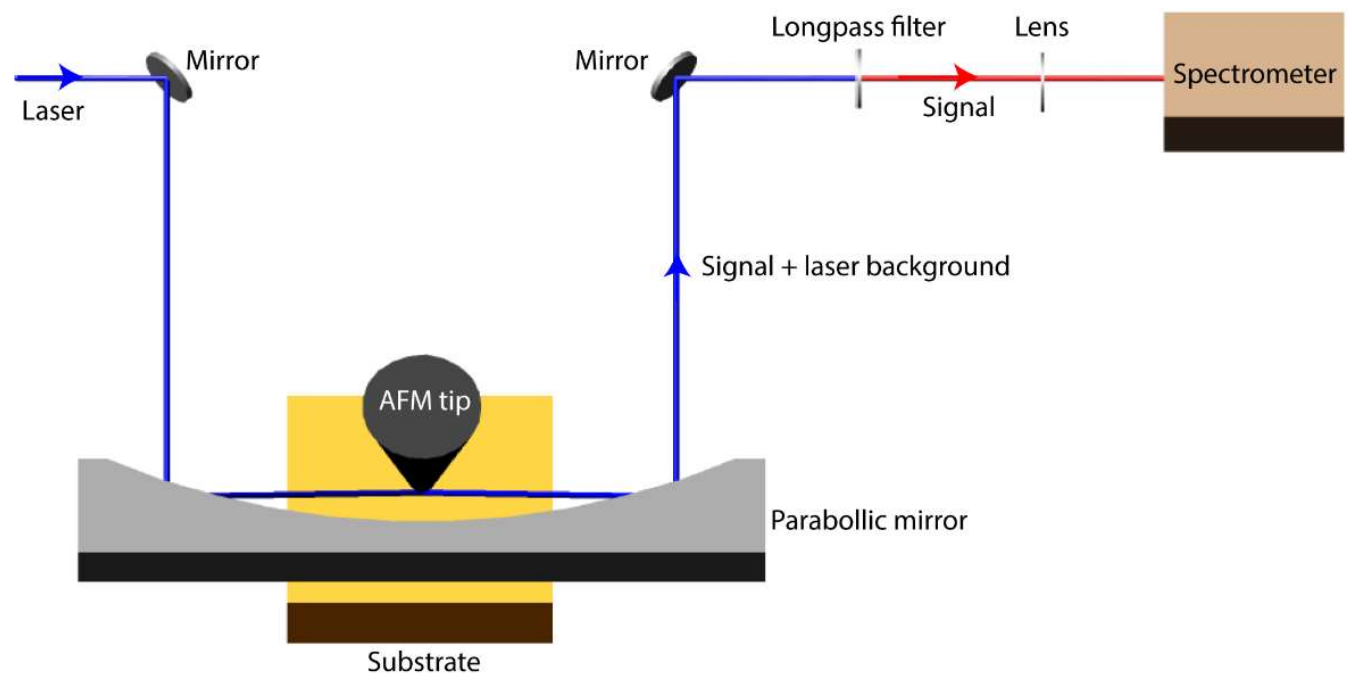

Figure S1 Schematic of the experimental setup to perform the SERS measurement. The laser is focused on the sample using a parabolic mirror at $60^{\circ}$ angle of incidence with respect to the normal to the substrate. The laser is vertically polarized but because of the grazing incidence the excitation field has both vertical and horizontal components. The scattered light is collected in the opposite direction and the reflected laser background is removed using longpass filter. The signal is then focused at the slit of the spectrometer using a lens with $10 \mathrm{~cm}$ focal length. 
(a) Gold nanospheres with CA surface ligand after treated in MB solution
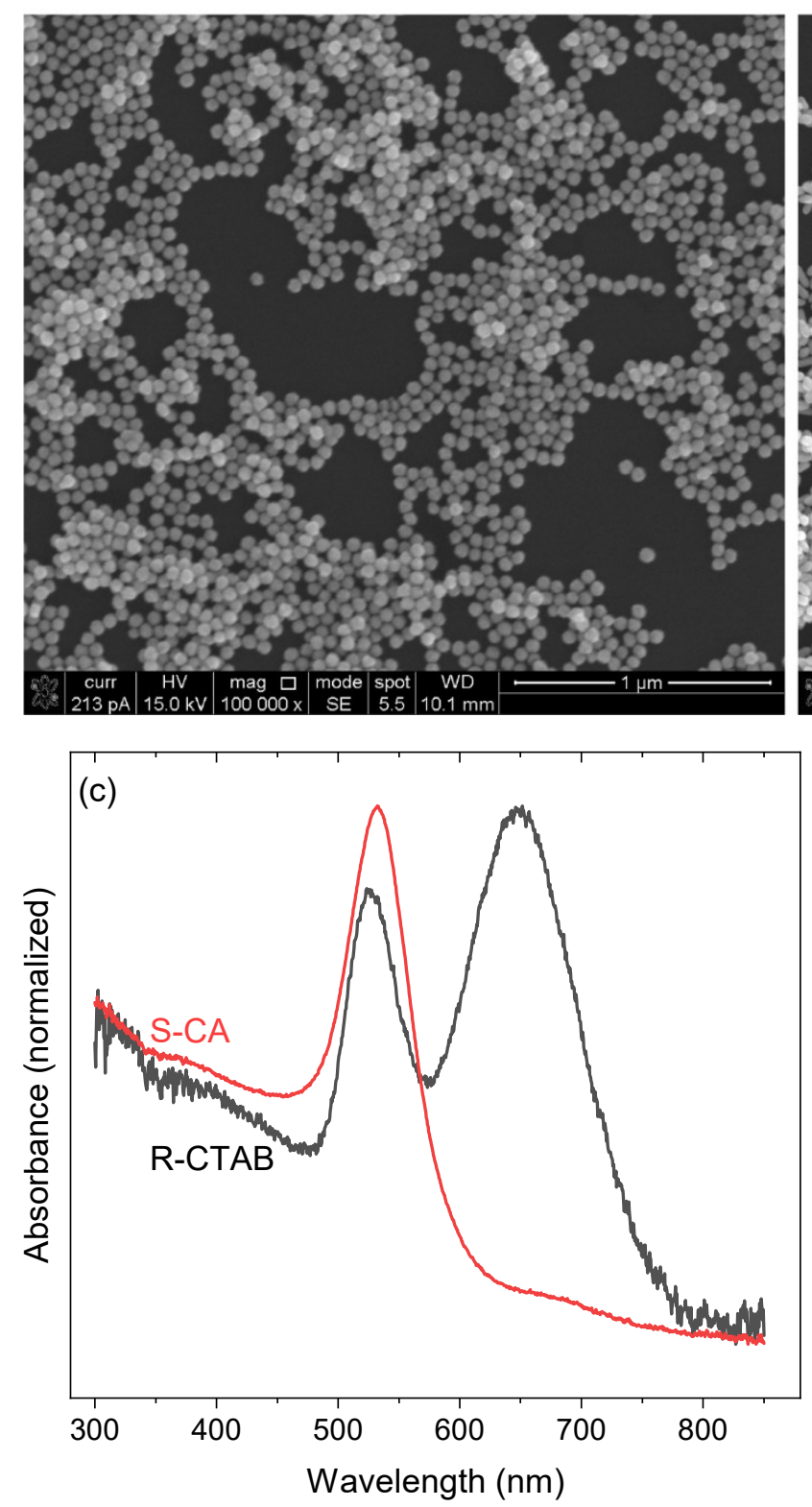

(b) Gold nanorods with CTAB surface ligand after treated in $\mathrm{MB}$ solution
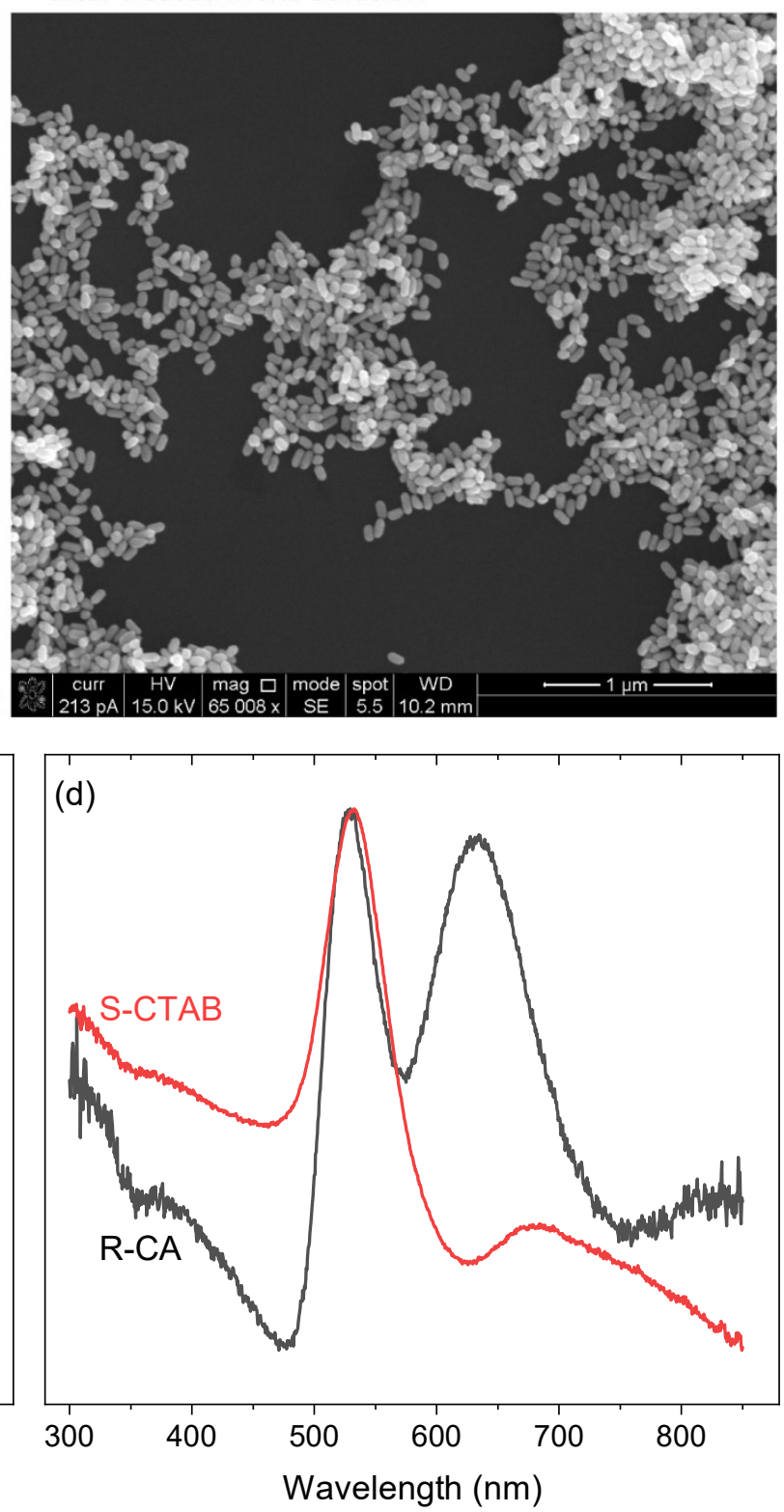

Figure S2 Scanning electron microscope (SEM) images of MB treated gold nanospheres (a) and gold nanorods (b). After the excess surfactant was removed, the gold nanoparticles were suspended in MB solution overnight. The MB/colloidal solution was then centrifuged and the supernatant solution was discarded. The solid residue was then re-suspended in ultrapure water and drop-casted on silicon wafer. The SEM images show the distribution on the wafer. (c) Absorption spectra of colloidal solutions of gold nanospheres (red line) and gold nanorods (black line). (d) Absorption spectra of colloidal solutions of gold nanospheres (red line) and gold nanorods (black line) after the surface ligands are exchanged. 


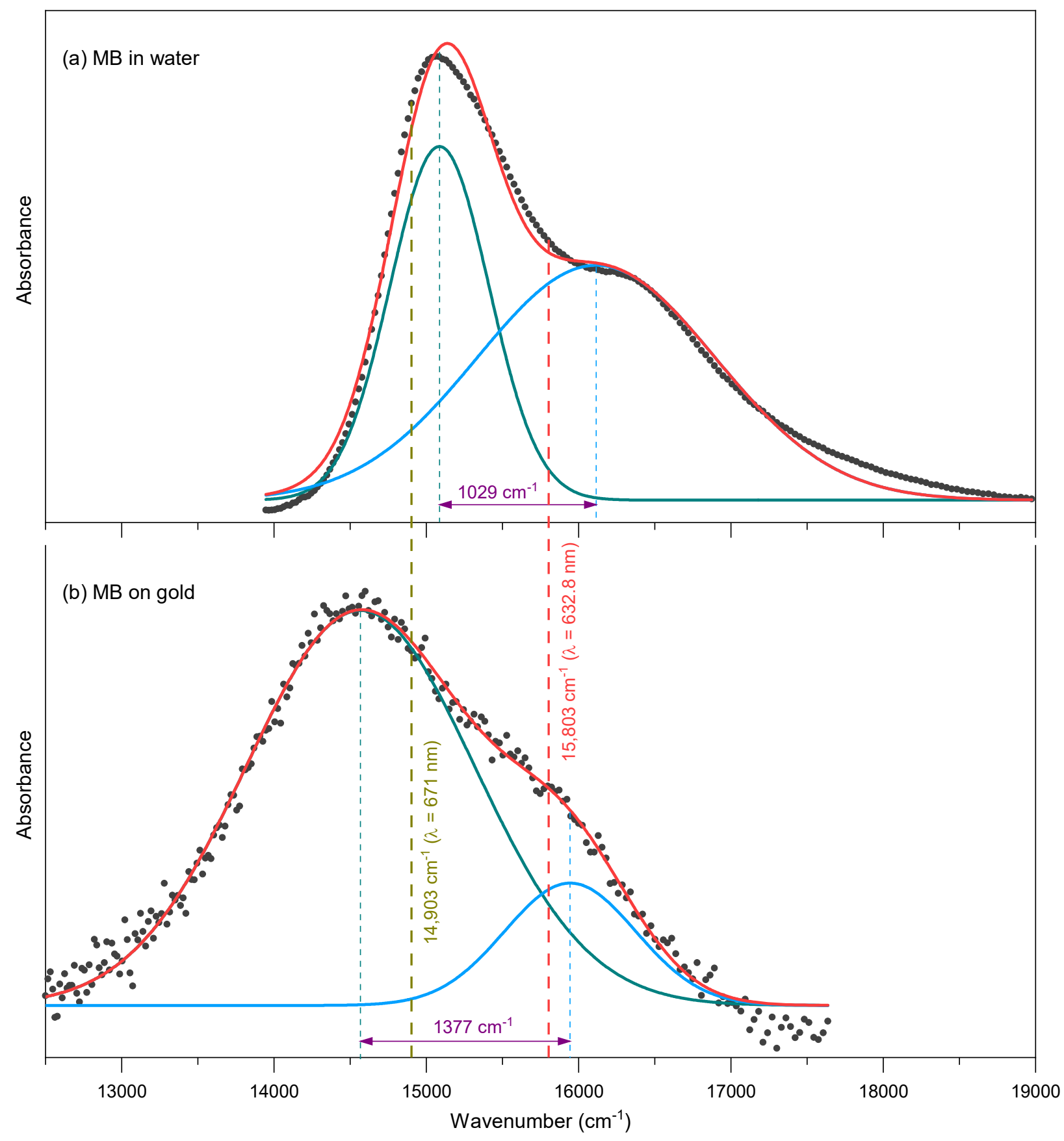

Figure S3 Absorption spectum of MB in water (a), and adsorbed on gold nanoislands (b). The absorption band of MB adsorbed on gold nanoislands is determined as described in our recent article (J. Phys. Chem. Lett. 2020, 11, 3507-3514). The x-axis is converted to wavenumber to facilitate the comparison with the transition energy in Figure 4 in the main text. Gaussian fitting is used to determine the center of the $0-0$ (dark cyan line) and $0-1$ (ligh blue line) transitions. The $0-1$ transition is accessible at $633 \mathrm{~nm}$ but not at $671 \mathrm{~nm}$. 


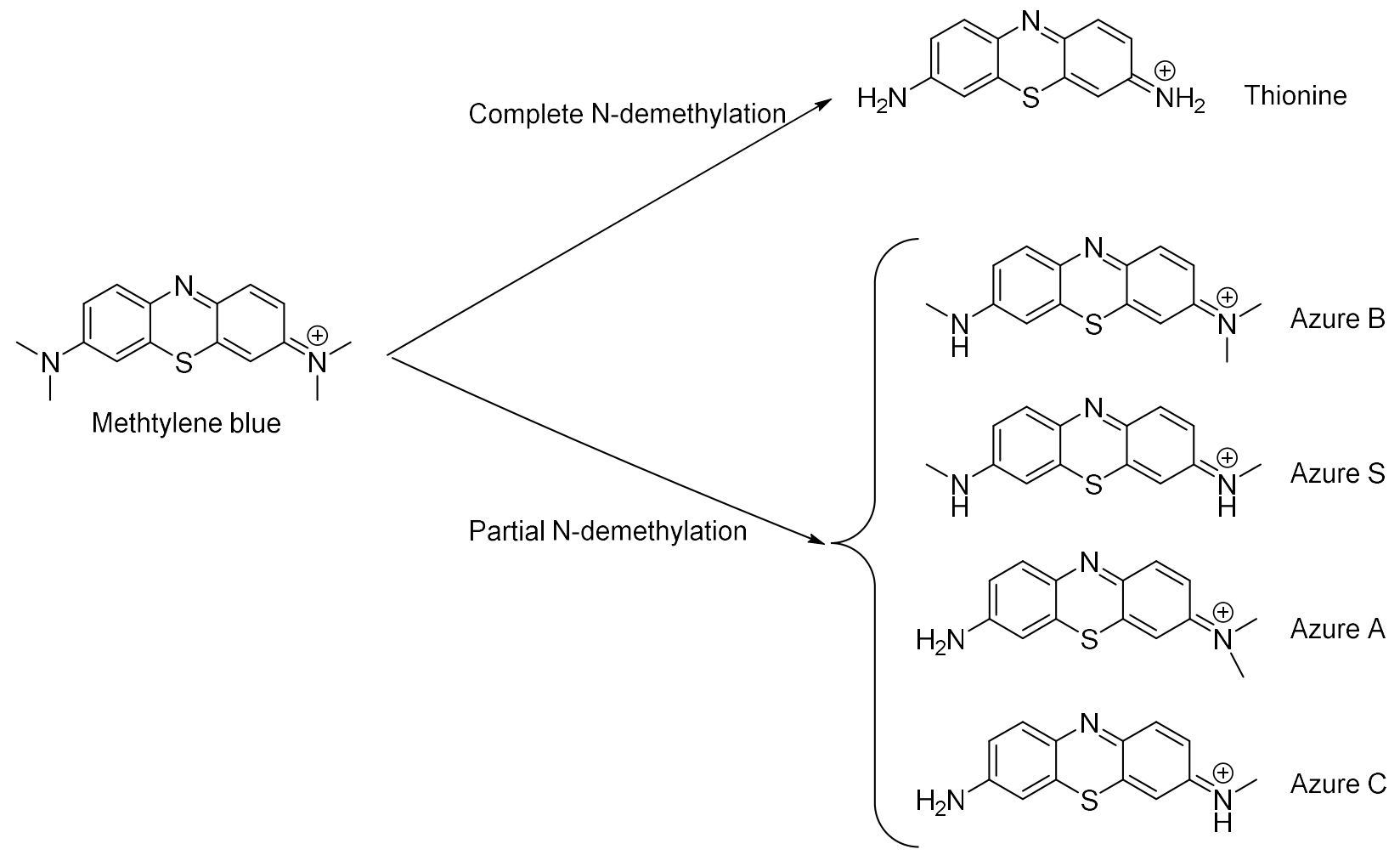

Scheme S1 


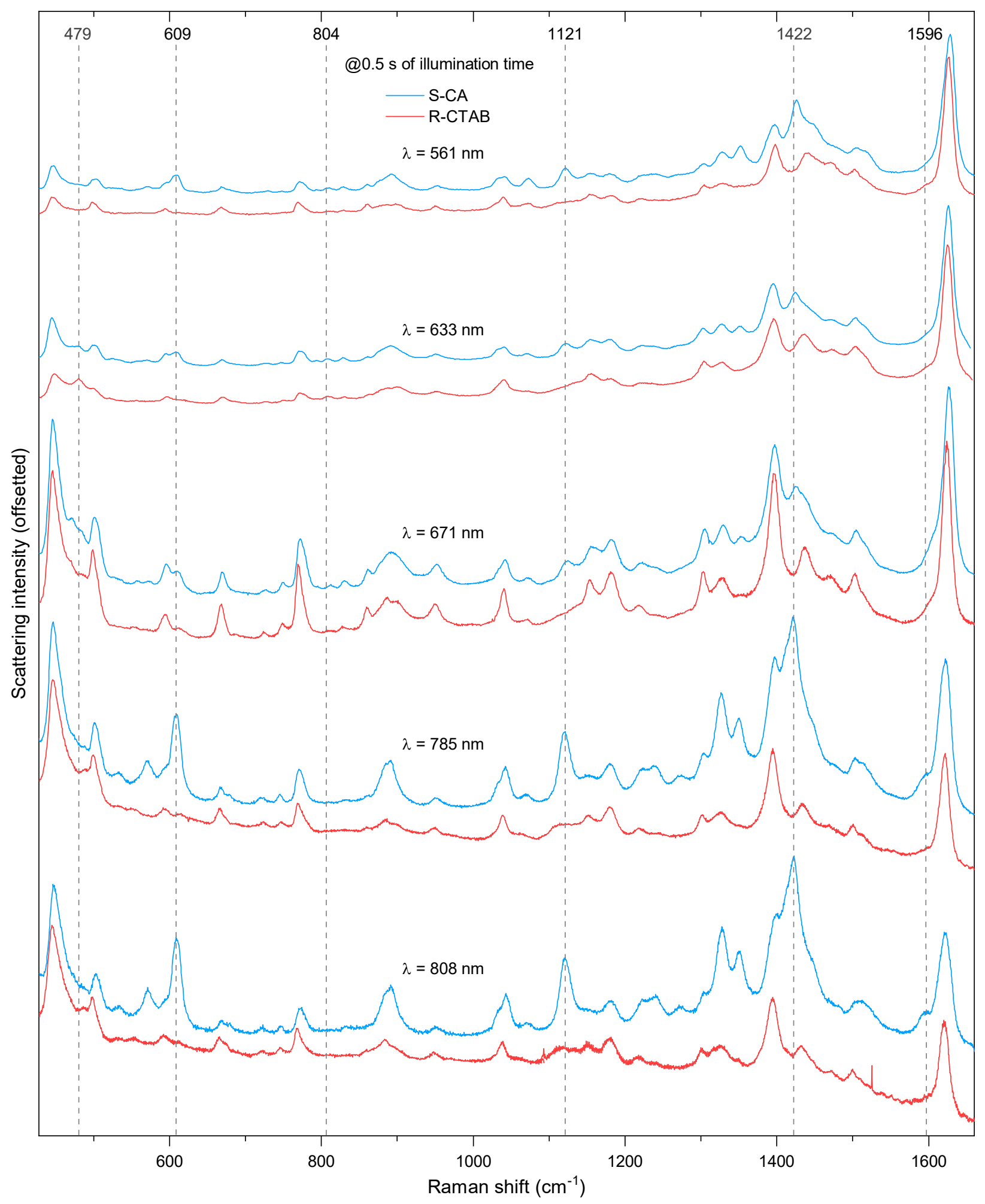

Figure S4a SERS spectra of MB adsorbed on S-CA (blue line) and on R-CTAB (red line) at different excitation wavelengths at $0.5 \mathrm{~s}$ acquistion time. That first spectra out of 200 represented in the intensity maps in Figure 2. 


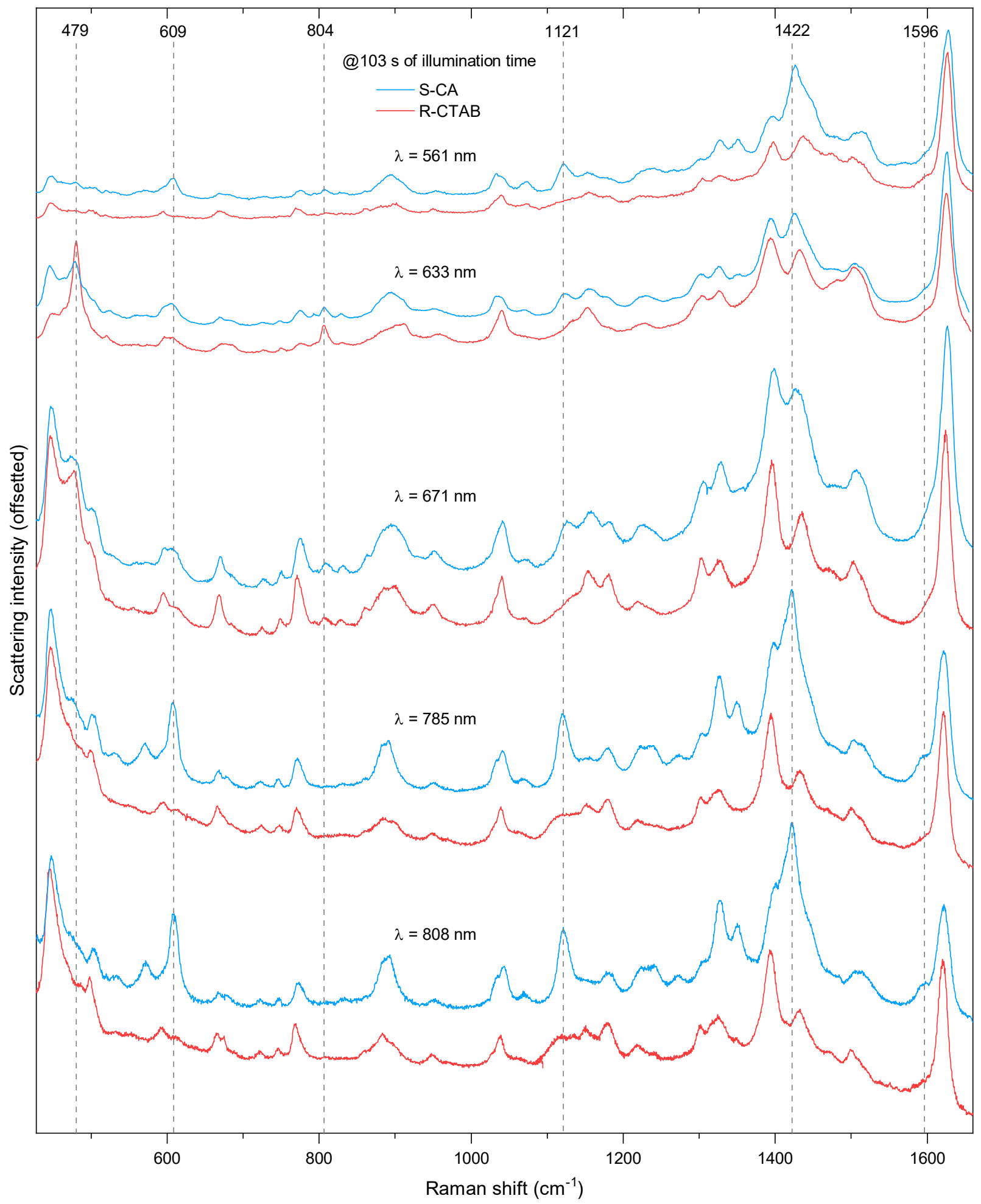

Figure S4b Same as Figures S4a but the last spectra recorded at 103 s of illumination time. 


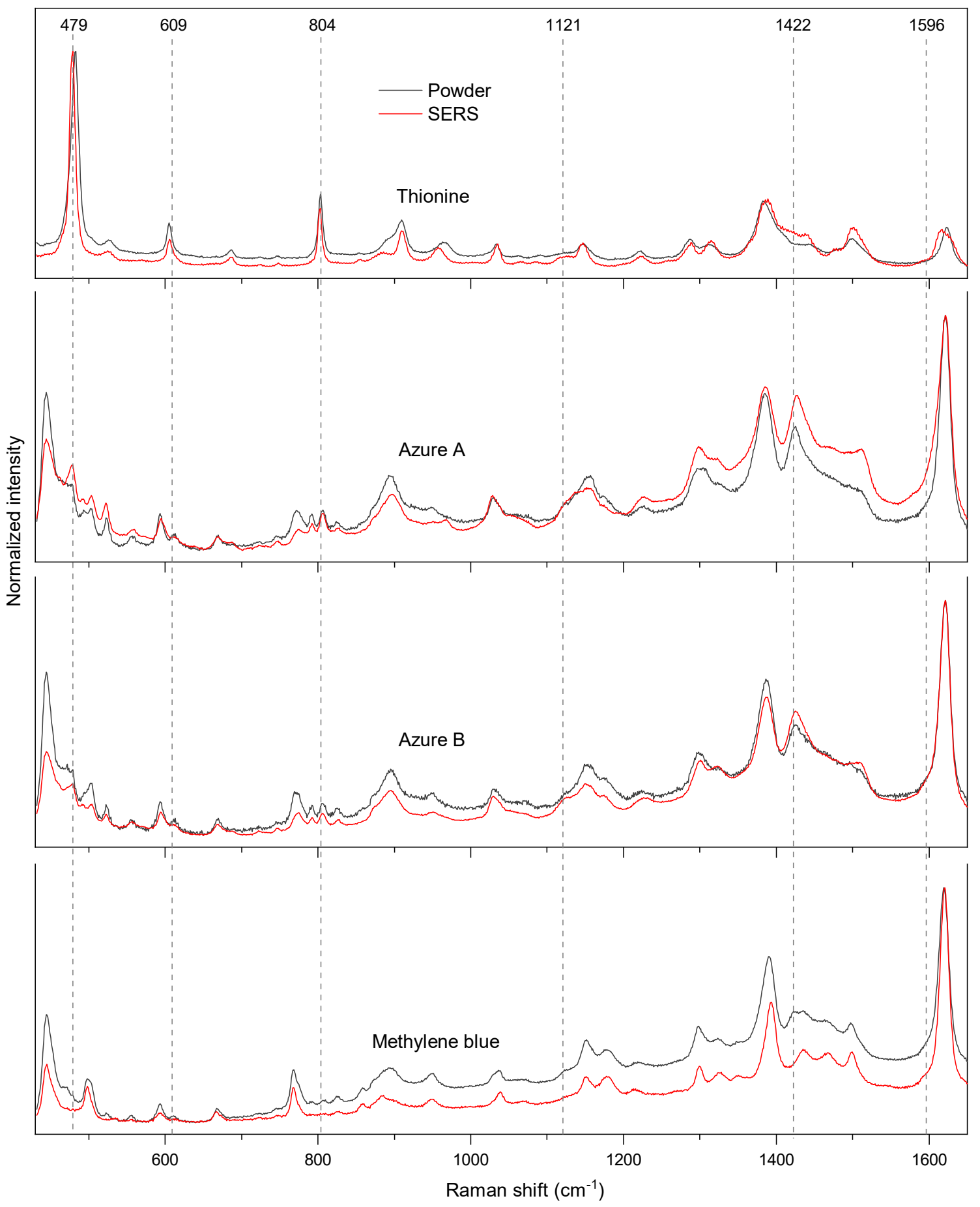

Figure S5 Normal (black lines) and SERS (red lines) spectra of methylene blue, azure B, azure A and thionine. The vertical dashed lines are included for facilitating the comparison with the SERS spectra in Figures 6 and S4. The peaks at $609 \mathrm{~cm}^{-1}$ and $1121 \mathrm{~cm}^{-1}$ are prominent in the spectra obtained at $808 \mathrm{~nm}$ and $785 \mathrm{~nm}$ for MB adsorbed on S-CA and bare gold nanoislands (see Figures 6 and S4). On the other hand, the $804 \mathrm{~cm}^{-1}$ peak that is prominent in the thionine spectrum and significant in azure A and azure B is completely absent in the SERS spectra obtained at $808 \mathrm{~cm}^{-1}$ and $785 \mathrm{~cm}^{-1}$. Other details can similarly be compared. 


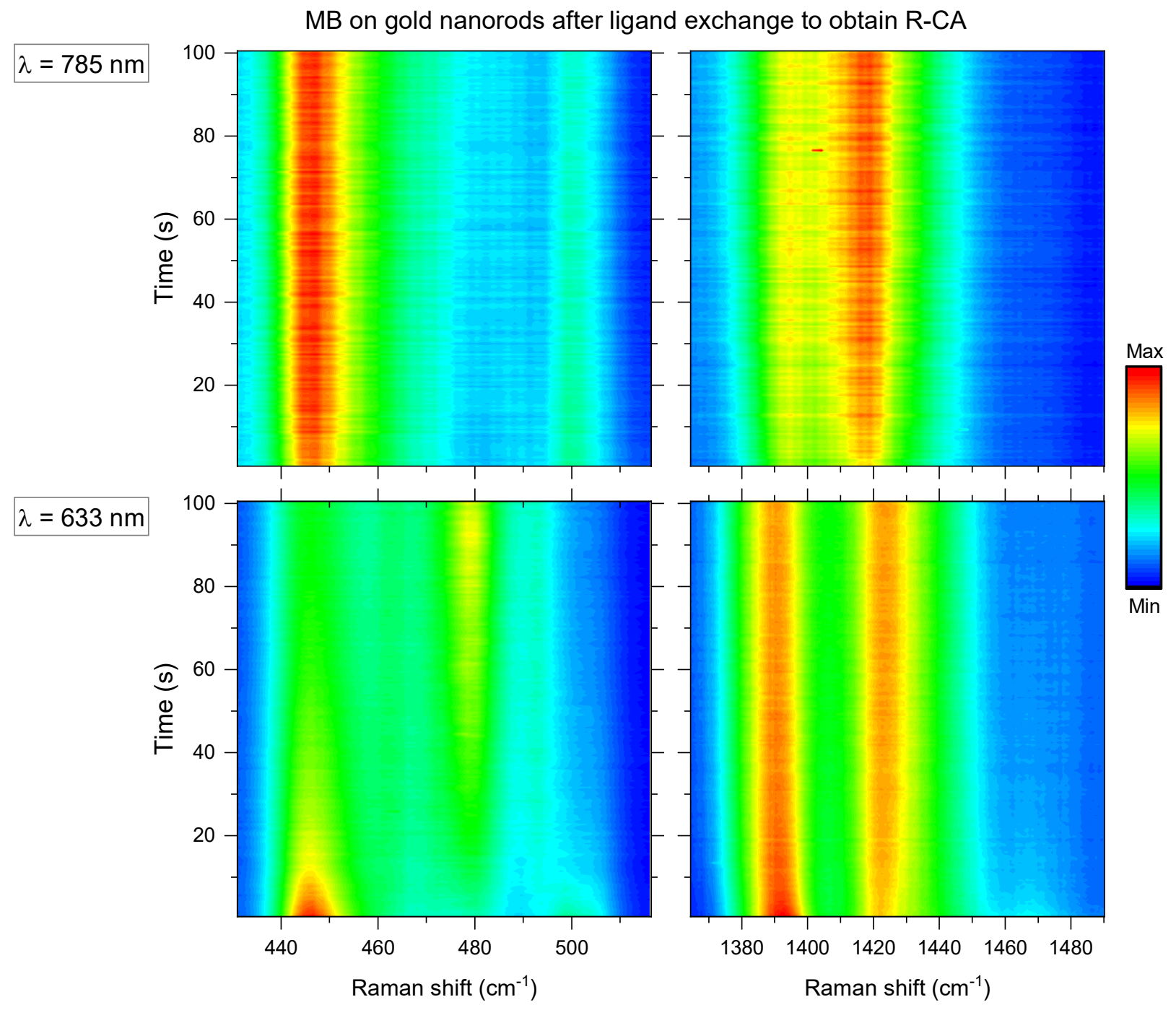

Figure S6 Intensity map of SERS spectra of MB adsorbed on R-CA (gold nanorods after the CTAB surface ligand is replaced with carboxylic acid) obtained at (a) $785 \mathrm{~nm}$, and (b) $633 \mathrm{~nm}$. Comparing with the results in Figure 2, it is clear that the patterns have strong similarity to the corresponding intensity maps for MB adsorbed on S-CA. 
(a)

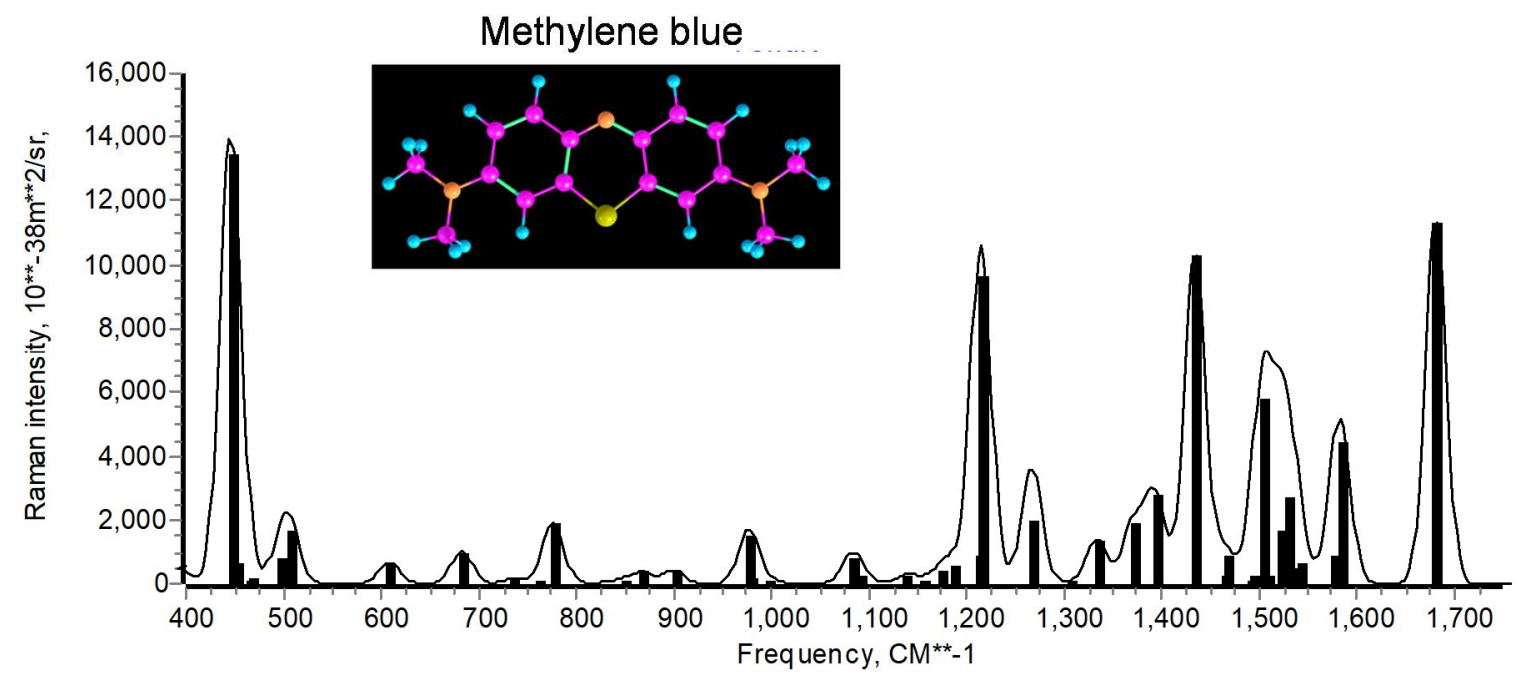

(b)

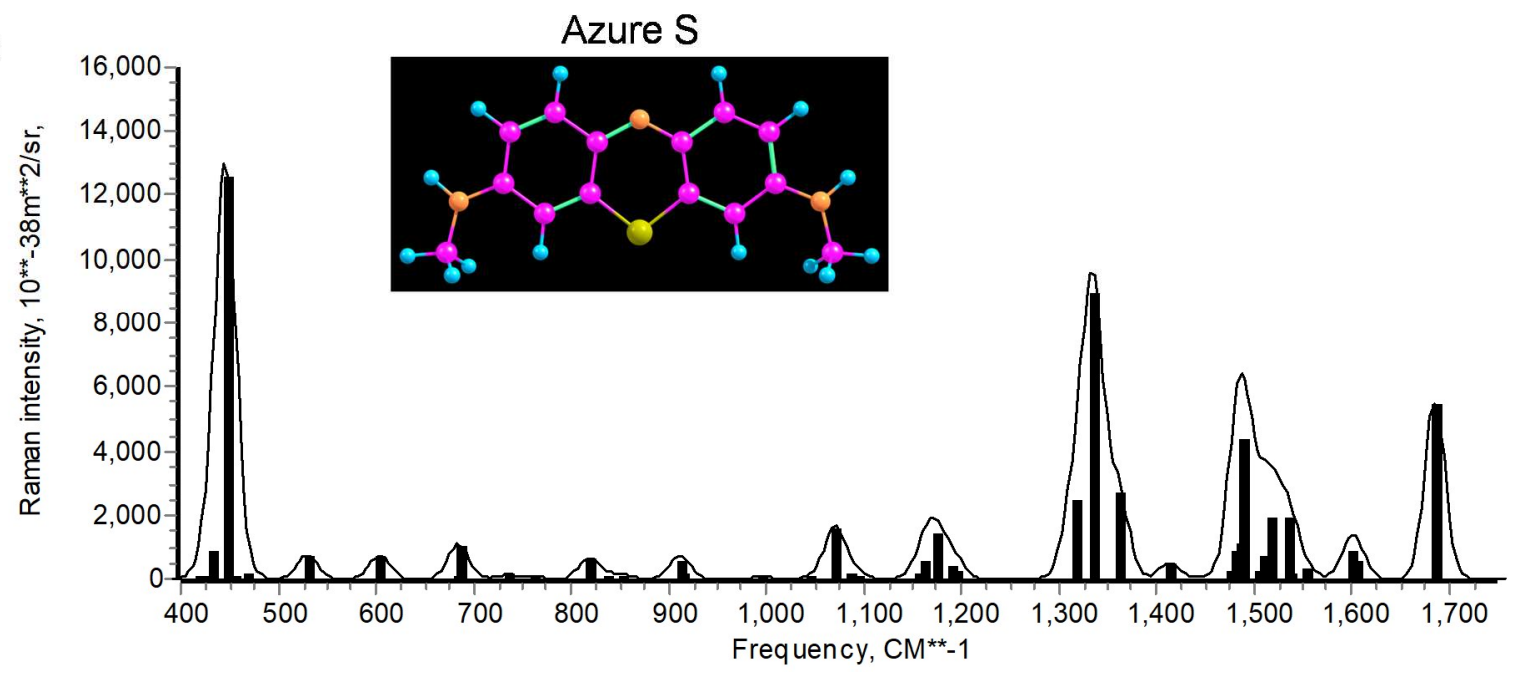

Figure S7 Calculated Raman spectra of (a) MB, and (b) azure S. The two spectra are calculated using the same method (B3LYP) and basis set (DGDZVP). The MB spectra has strong similarity to the experimentally observed spectra except that the calculated peak positions in the high frequency region overestimates the observed values. We suspected the spectra observed at 808 and $785 \mathrm{~nm}$ for $\mathrm{MB}$ adsorbed directly on gold could be assigned to azure $\mathrm{S}$ but the calculated spectra in (b) does not support this assignment, and it is therefore ruled out. 

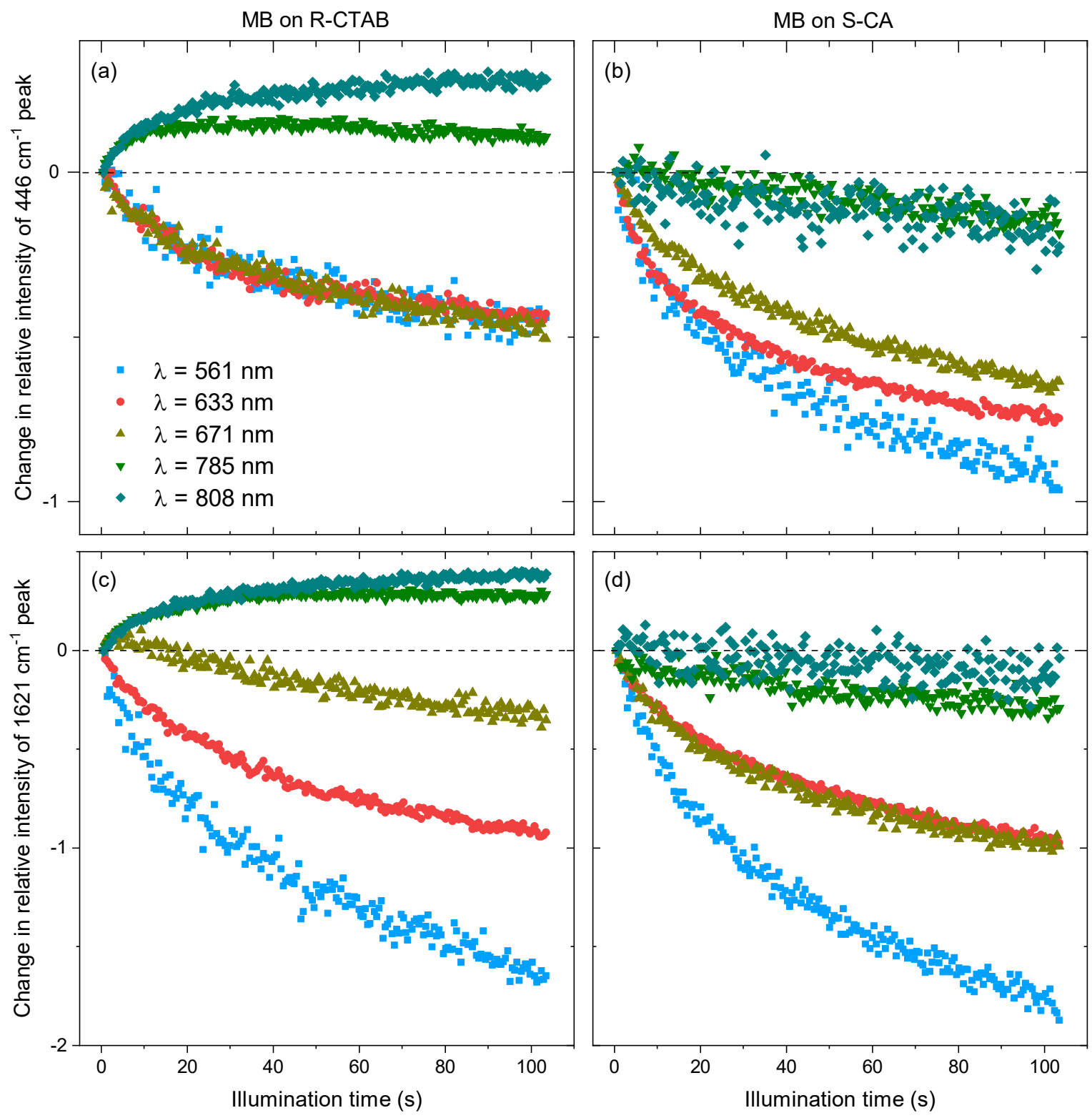

Figure S8 Change in relative peak intensity of representative modes as a function of illumination time: (a, b) for the $446 \mathrm{~cm}^{-1}$ mode, and (c, d) for $1621 \mathrm{~cm}^{-1}$ mode. Both of these peaks appear in all MB derivatives except thionine for which the former does not exist and later is weak as shown in Figure S5. The fact that the relative intensity of both peaks are increasing with time during illumination of MB adsorbed on R-CTAB at $808 \mathrm{~nm}$ and $785 \mathrm{~nm}$, at which no chemical transformation is observed, indicates chemical enhancement contribution to the SERS signal. The chemical enhancement can originate from plasmon induced reorganization of adsorption geometry that leads to improved surface-molecule interaction. 